\title{
Tailoring Membrane Surface Charges: A Novel Study on Electrostatic Interactions during Membrane Fouling
}

\author{
Daniel Breite ${ }^{1}$, Marco Went ${ }^{1}$, Andrea Prager ${ }^{1}$ and Agnes Schulze ${ }^{1, *}$ \\ Received: 7 August 2015 ; Accepted: 9 October 2015 ; Published: 19 October 2015 \\ Academic Editor: Scott M. Husson \\ Leibniz Institute of Surface Modification, Permoserstr. 15, 04318 Leipzig, Saxony, Germany; \\ daniel.breite@iom-leipzig.de (D.B.); marco.went@iom-leipzig.de (M.W.); andrea.prager@iom-leipzig.de (A.P.) \\ * Correspondence: agnes.schulze@iom-leipzig.de; Tel.: +49-341-2352-400; Fax: +49-341-2353-293
}

\begin{abstract}
In this work we aim to show that the overall surface potential is a key factor to understand and predict anti-fouling characteristics of a polymer membrane. Therefore, polyvinylidene fluoride membranes were modified by electron beam-induced grafting reactions forming neutral, acidic, alkaline or zwitterionic structures on the membrane surface. The differently charged membranes were investigated regarding their surface properties using diverse analytical methods: zeta potential, static and dynamic water contact angle, scanning electron microscopy (SEM) and X-ray photoelectron spectroscopy (XPS). Porosimetry measurements proved that there is no pore blocking due to the modifications. Monodisperse suspensions of differently charged polystyrene beads were synthesized by a radical emulsion polymerization reaction and were used as a model fouling reagent, preventing comparability problems known from current literature. To simulate membrane fouling, different bead suspensions were filtered through the membranes. The fouling characteristics were investigated regarding permeation flux decline and concentration of model fouling reagent in filtrate as well as by SEM. By considering electrostatic interactions equal to hydrophobic interactions we developed a novel fouling test system, which enables the prediction of a membrane's fouling tendency. Electrostatic forces are dominating, especially when charged fouling reagents are present, and can help to explain fouling characteristics that cannot be explained considering the surface wettability.
\end{abstract}

Keywords: PVDF membrane; grafting; fouling; polystyrene bead; electrostatic interaction

\section{Introduction}

\subsection{Membrane Surface Hydrophilicity and Surface Charge}

Membrane fouling is a problem that scientists have tried to counter for decades. Because membranes are always requested to be more durable, solvent resistant, and long-term stable, most of the polymers used for membrane production are highly hydrophobic. However, this hydrophobicity leads to hydrophobic interactions with, e.g., proteins resulting in a fouling layer precipitated on the membrane surface accompanied by a decreased membrane performance [1,2]. The common way to face this problem is to hydrophilize the hydrophobic membrane material [3-10] with different modification methods like grafting reactions [6,8-12], electron beam (EB) irradiation $[13,14]$ or plasma treatment [5,15-20]. The hydrophilized membrane surfaces then show an increased fouling resistance due to the buildup of a water film on the membrane surface, which repels hydrophobic fouling reagents [21]. The general agreement was and is that the most hydrophilized membrane surface exhibits the highest fouling resistance. In any case, other thinkable interactions between membranes 
and fouling reagents like hydrogen bonds, electrostatic or dipolar forces, which will also be altered with the above named methods, were rarely taken into consideration. However, there are just a few studies that show the role of a membrane's surface charge and therefore possible electrostatic interactions while membrane fouling, but the research interest in this direction is increasing recently. Kumar and Ulbricht [22] for example showed the improved anti-fouling characteristics of membranes depending on the charge of the proteins filtered through, and how this can be utilized to gain selective permeability. Xiao et al. [23] presented a semi-empirical model, which uses zeta potentials to determine fouling tendencies, and Hadidi and Zydney [24] showed the effect of using differently charged membranes for fouling with differently charged proteins. They found reduced fouling for combinations of similar charges and also presented the unique characteristics of zwitterionic structures, which seem to have good anti-fouling properties even in the case of opposite charges. Other works by Zhang et al. [25] or Dudchenko et al. [26] showed how the application of an external electrical potential impacts the fouling of carbon nanotube-polyvinylidene fluoride (PVDF) membranes. Elimelech et al. did extensive work on the influence of electrostatic interactions to the fouling with natural organic matter $[27,28]$.

\subsection{Description of Fouling Tendency}

As mentioned above, the marker for the fouling tendency so far is the membrane's surface hydrophilicity characterized by the water contact angle. The problem here is that, following Young's equation, the contact angle depends on the surface energy, which again depends on the surface area [29]. This brings up the issue of membrane surface roughness and makes water contact angles difficultly comparable when the surface roughness is also altered due to membrane modification. A smarter way to describe a membrane's fouling tendency is to measure the surface potential. The surface potential itself is experimentally inaccessible but an approximate surface potential can be measured by electrokintetic measurements. The so-called zeta potential describes the surface potential of the modified surface at the shear plane while in contact with an electrolyte, and by plotting the zeta potential against electrolyte characteristics like $\mathrm{pH}$, knowledge about the membrane surface charge under various conditions can be gained.

\subsection{Polystyrene Beads as Model Fouling Reagent}

Another issue of comparability is the choice of the fouling reagent. Model fouling reagents often described in literature like proteins, humic acids, natural organic matter, or different polysaccharides are used in individual mixtures and concentrations, and are therefore hard to compare. Furthermore, a prediction for the fouling tendencies in real systems is nearly impossible. Proteins for example tend to unfold and lose their active structure while fouling a membrane [30]. This can lead to false assumptions for the comparison of different fouling experiments, even if the compared proteins had nearly the same size and shape in the first place. Therefore, a uniform fouling reagent with tunable surface charge is essential to describe the role of electrostatic interaction while membrane fouling, e.g., polystyrene (PS) beads. As already described by several groups, PS beads can be used as model fouling reagent [31-44]. Nevertheless, an investigation regarding the fouling of differently charged PS beads is missing so far. The nearly ideal spherical beads can be easily synthesized by a radical emulsion polymerization while tuning their surface charge by the choice of initiator. The bead size can also be tuned to the desired value by a variation of reaction parameters. This way membrane fouling can be described using differently charged PS beads of the same size to maintain comparability of the different fouling experiments.

By considering electrostatic repulsive and attractive forces equal to hydrophobic interactions, we developed a novel fouling test system that shows that electrostatic forces can help to understand fouling, especially when it is unexpected and cannot be explained considering the membrane's surface wettability. 


\section{Experimental Section}

\subsection{Reagents and Materials}

Used PVDF flat sheet membranes were obtained from Carl Roth (Roti-PVDF, $0.45 \mu \mathrm{m}$, Karlsruhe, Germany). The following chemicals were obtained from Sigma Aldrich (St. Louis, MS, USA): styrene, potassium persulfate (KPS), 2,2'-azobis(2-methylpropionamidine) dihydrochloride (AIBA), 2,2'-azobis(2-methylpropionitrile) (AIBN), lauryldimethylammonia acetat, tetraethylpentamine (TEPA), lysine, polyethylene glycol (PEG, $3350 \mathrm{~g} / \mathrm{mol}$ ), polystyrene sulfonate (PSS, 70,000 g/mol). Other purchased chemicals: aluminum oxide (Brockmann activity I, Fluka, Sigma Aldrich, St. Louis, MS, USA), n-hexane (Merck, Darmstadt, Germany), 2-aminoethyl methacrylate hydrochloride (AEMA, Acros Organics, Thermo Fisher Scientific, Geel, Belgium), glutaraldehyde (Merck, Darmstadt, Germany), hydrochloric acid solution (0.1 M, VWR, Radnor, PA, USA), sodium hydroxide solution (0.1 M, VWR, Radnor, PA, USA), sodium carbonate (anhydrate, VWR, Radnor, PA, USA), sodium bicarbonate (Thermo Fisher Scientific, Geel, Belgium). All chemicals named above are used as received. The used water was ultrapure water taken from a MilliQ-System (Billerica, MA, USA). Dialysis membranes used for particle purification were obtained from Carl Roth (cellulose acetate, Nadir, molecular weight cut-off (MWCO): 10,000-20,000 Da, Wiesbaden, Germany).

\subsection{Synthesis and Characterization of Differently Charged PS Beads}

PS beads were synthesized using a liquid phase synthesis method, involving a dispersion polymerization of styrene monomer in aqueous solution. The used styrene monomer was purified before usage by passing it though an aluminum oxide column to remove the polymerization inhibitor. For synthesis, water was filled into the reaction system composed of a three-necked flask equipped with a reflux condenser, a mechanical stirrer, and a septum. The system was heated to and kept at $70{ }^{\circ} \mathrm{C}$ by an oil bath, and kept under nitrogen atmosphere until the end of the reaction time. Styrene monomer was added to the heated and degased reaction system via a syringe to gain a $5 \mathrm{wt} \%$ emulsion. The reaction was started by addition of the initiator $(8 \mathrm{mmol} / \mathrm{L}$ in final solution). For the synthesis of anionic PS beads, KPS [45] was chosen as initiator, for the synthesis of cationic PS beads, AIBA [46] was chosen, each dissolved in $5 \mathrm{~mL}$ water for addition via syringe. In the case of uncharged PS beads, the initiator AIBN [47] was dissolved in $5 \mathrm{~mL}$ of detained styrene monomer and added via syringe. After $30 \mathrm{~min}$ reaction time, the surfactant lauryldimethylammonia acetate $(0.5 \mathrm{mmol} / \mathrm{L}$ in final solution) was added via syringe, also dissolved in $5 \mathrm{~mL}$ water. At the end of the reaction time, the system was vented with air, and the gained particle suspension was washed with $n$-hexane to remove leftover monomer. The final suspension was then dialyzed three times using a dialysis membrane and MilliQ ultrapure water. The precise conditions for the synthesis of the different PS beads are listed in Table 1.

Table 1. Synthesis parameters for differently charged polystyrene (PS) beads.

\begin{tabular}{cccc}
\hline Particle charge & Initiator & Impeller speed (rpm) & Time (h) \\
\hline positive & AIBA & 200 & 5 \\
negative & KPS & 400 & 2 \\
uncharged & AIBN & 200 & 2 \\
\hline
\end{tabular}

The differently charged PS particle suspensions were characterized using the Malvern Zetaziser (Zetasizer Nano ZS with multipurpose titrator MPT-2, Malvern Instruments, Worcestershire, UK) to determine particle size, zeta potential, and isoelectric point (IEP). Scanning electron microscopy (SEM) pictures (Ultra 55 SEM, Carl Zeiss Ltd., Goettingen, Germany) were taken from particles spin-coated on a silica wafer. 


\subsection{Membrane Modification and Characterization}

For grafting modification the membranes were dipped into an aqueous solution of PEG $(0.5 \mathrm{wt} \%)$ [48] or PSS ( $2 \mathrm{wt} \%)$ [48], followed by EB irradiation in wet state. Irradiation was performed in a $\mathrm{N}_{2}$ atmosphere with $\mathrm{O}_{2}$ quantities $<10 \mathrm{ppm}$ using a custom-made electron accelerator and an irradiation dose of $200 \mathrm{kGy}$. The voltage and the current were set to $160 \mathrm{kV}$ and $10 \mathrm{~mA}$, respectively. The absorbed dose was adjusted by the speed of the sample transporter.

To generate an alkaline membrane surface the membranes were dipped into an aqueous solution of AEMA (0.5 wt \%), followed by EB irradiation (150 kGy) and rinsing with water three times for $30 \mathrm{~min}$. Then, the membranes were dipped into an aqueous solution of GA ( $2 \mathrm{wt} \%)$ at $\mathrm{pH}$ $9.2\left(\mathrm{NaHCO}_{3} / \mathrm{Na}_{2} \mathrm{CO}_{3}\right.$ buffer system) for $2 \mathrm{~h}$. The GA solution was removed and the membrane was roughly rinsed before it was immersed into an aqueous solution of TEPA ( $2 \mathrm{wt} \%$ ) at $\mathrm{pH} 9.2$ $\left(\mathrm{NaHCO}_{3} / \mathrm{Na}_{2} \mathrm{CO}_{3}\right.$ buffer system) for another $2 \mathrm{~h}$. The reactions with GA and TEPA were repeated as described before to create a 2nd generation of dendrimeric structures [49].

To generate zwitterionic structures the membranes were dipped into an aqueous solution of AEMA ( $2 \mathrm{wt} \%)$, followed by EB irradiation ( $200 \mathrm{kGy}$ ) and rinsing with water three times for $30 \mathrm{~min}$. The subsequent reaction with GA was performed as described for the alkaline modification, but in the next step instead of TEPA lysine (2 $\mathrm{wt} \%)$ was used.

Finally, all modified membranes were rinsed three times for $30 \mathrm{~min}$ and subsequently dried at room temperature.

The morphology of the modified membranes was characterized using SEM imaging (Ultra 55 SEM, Carl Zeiss Ltd., Goettingen, Germany), where the samples were coated with a thin $(30 \mathrm{~nm})$ chromium film using a Z400 sputter system (Leybold, Hanau, Germany). Pore size distribution and porosity of the membranes were determined with a mercury porosimeter (PoreMaster 30, Quantachrome Instruments, Odelzhausen, Germany). Values of at least three different samples were averaged. Water permeability was investigated using a stainless steel pressure filter holder (16249, Sartorius, Goettingen, Germany) for dead end filtration. A volume of $200 \mathrm{~mL}$ of deionized water was passed through the membrane sample (active area: $17.3 \mathrm{~cm}^{2}$ ) at 1 bar and the time of flow-through was recorded. Values of at least three different samples were averaged. Pure water permeation flux $J$ was calculated following Equation (1).

$$
J=\frac{V}{t \cdot A \cdot p}
$$

where $V$ is the volume of deionized water passed through the membrane, $t$ the time of flow-through, $A$ the active area of the membrane sample and $p$ the applied pressure. The chemical composition was determined using X-ray photoelectron spectroscopy (XPS, Kratos Axis Ultra, Kratos Analytical Ltd., Manchester, UK). The membrane's surface wettability was investigated using a static and dynamic water contact angle measurements system (DSA 30E, Krüss, Hamburg, Germany) with the sessile drop method. The drop soaking rate was determined based on the change in water contact angle within the first $15 \mathrm{~min}$. Values of at least three different samples were averaged. Membrane zeta potentials were determined using streaming potential measurements carried out with the adjustable gap cell in the SurPASS system (Anton Paar, Graz, Austria), where the zeta potential $\zeta$ can be calculated based on the Smoluchowski equation given in Equation (2). Values of at least three different samples were averaged.

$$
\zeta=\frac{d U}{d p} \cdot \frac{\eta}{\varepsilon \cdot \varepsilon_{0}} \cdot \kappa
$$

where $U$ is the streaming potential, $p$ the pressure, $\eta$ the viscosity of the electrolyte solution, $\varepsilon$ the dielectric constant of the electrolyte solution, $\varepsilon_{0}$ the vacuum permittivity and $\kappa$ the electrolyte conductivity. 


\subsection{Membrane Fouling with PS Beads}

To determine the membrane fouling, the filtration experiments with PS beads were carried out in dead-end filtration mode using a $50 \mathrm{~mL}$ stirring cell (Amicon, Merck Millipore, Billerica, MA, USA). The membrane sample (active area: $15.9 \mathrm{~cm}^{2}$ ) was pre-wetted with ethanol for $1 \mathrm{~min}$ and subsequently washed with water for 5 min twice. Then, the sample was mounted into the stirring cell and a volume of $140 \mathrm{~mL}$ water was passed through the membrane at 0.1 bar to check water permeability and make sure that no pore blocking occurred due to the membrane modification. Afterwards, a volume of $140 \mathrm{~mL}$ PS bead suspension $(\sim 50 \mathrm{mg} / \mathrm{L})$ at $\mathrm{pH} 7$ was passed through the membrane under the same conditions, and the filtrate fractions were taken after every $20 \mathrm{~mL}$. In the case of membranes modified with zwitterionic structures, PS bead suspensions at $\mathrm{pH} 4$ or 9 were also filtered through the membranes. The $\mathrm{pH}$ of every suspension was checked prior to use with a pH electrode system (HI 3220, Hanna Instruments, Kehl, Germany). To adjust the $\mathrm{pH}$ to 4 or 9, hydrochloric acid solution $(0.1 \mathrm{M})$ or sodium hydroxide solution $(0.1 \mathrm{M})$ were used, respectively. The permeation flux decline was determined by recording the time of flow-through for each fraction. The concentration of PS beads was measured spectrometrically (Infinite M200, Tecan, Männedorf, Switzerland) using the UV absorption of polystyrene at $290 \mathrm{~nm}$. The absorption for every starting particle suspension was checked prior to every filtration experiment. Values of at least three different samples were averaged.

\section{Results and Discussion}

\subsection{Characteristics of PS Beads with Different Surface Charge}

The synthesis of PS particles, as described in Section 2.2, enables tuning of the surface charge of the desired beads. Anionic, cationic and uncharged particles were prepared to investigate the influence of electrostatic interactions that occur while the beads are filtered through a membrane.

Figure 1 shows the $\mathrm{pH}$ dependence of the particle's zeta potential. It is obvious that the particles formed with KPS as initiator possess an IEP of 2.5 (acidic/anionic) and are highly negatively charged $(-90 \mathrm{mV}$ at $\mathrm{pH} 7)$ due to the sulfate groups. Particles formed with AIBA have an IEP of 10.8 (alkaline/cationic) and are highly positive charged $(+74 \mathrm{mV}$ at $\mathrm{pH} 7)$ due to the amino groups. The uncharged species possess an IEP of around 3 and are slightly negatively charged ( $-32 \mathrm{mV}$ at $\mathrm{pH} 7$ ). This is due to the fact that even inert and uncharged surfaces have a small zeta potential resulting from adsorption of hydroxide ions from the self-ionization of water [50,51].

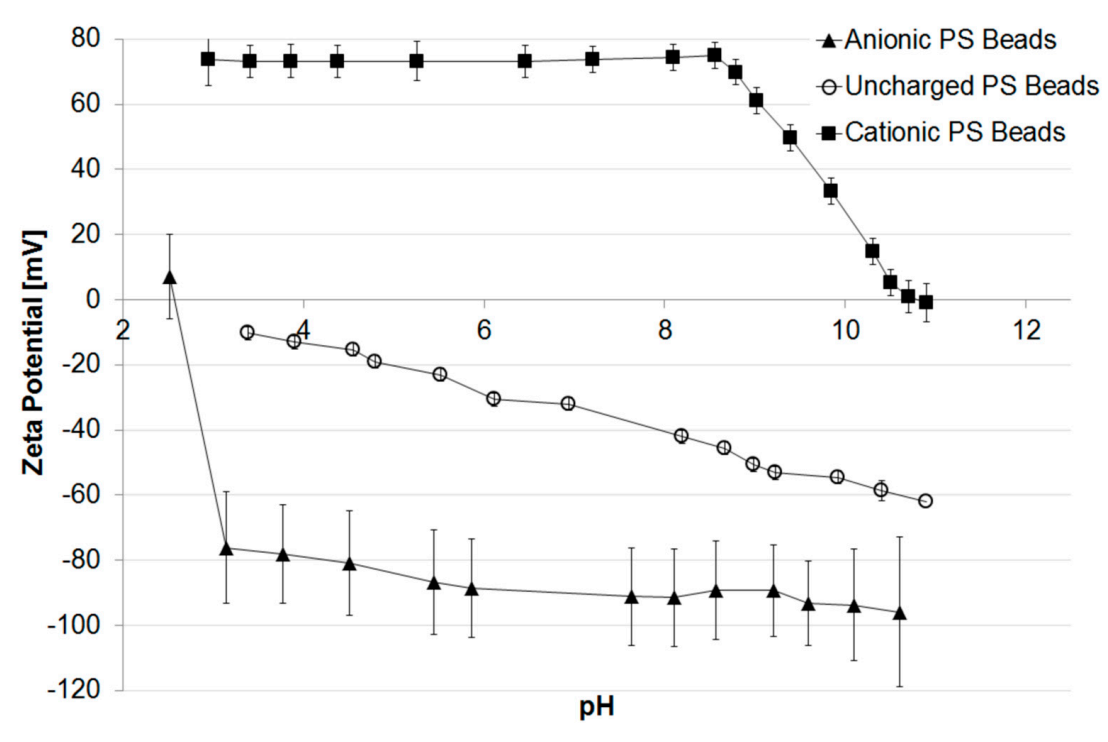

Figure 1. Zeta potential of differently charged PS beads vs. $\mathrm{pH}$. 
The particles are grown to a diameter of around $0.2 \mu \mathrm{m}$, which is smaller than the pore size of the PVDF membrane $(0.45 \mu \mathrm{m})$. Therefore, particles can pass through the membrane when no interactions between membrane and particles occur, and fouling due to size exclusion is prevented.

Further descriptions of the polymerization reaction mechanisms, the structures of the different initiator molecules and data regarding the particle size and dispersity are given in the Supplementary Material (Figures S1 and S2).

\subsection{Characteristics of Modified PVDF Membranes}

To investigate the influence of electrostatic interactions while membrane fouling, not only differently charged fouling reagents are needed, but also differently charged membranes. Therefore, modifications with anionic, cationic, uncharged (hydrophilic) or zwitterionic functionalities were applied to the PVDF membranes.

The modifications, as described in Section 2.3 with the reagents shown in Figure 2, enables to permanently hydrophilize a membrane's surface. Due to the electron beam irradiation radical species are formed, which lead, following a grafting reaction, to a chemical bonding between the membrane and the used modification reagent.
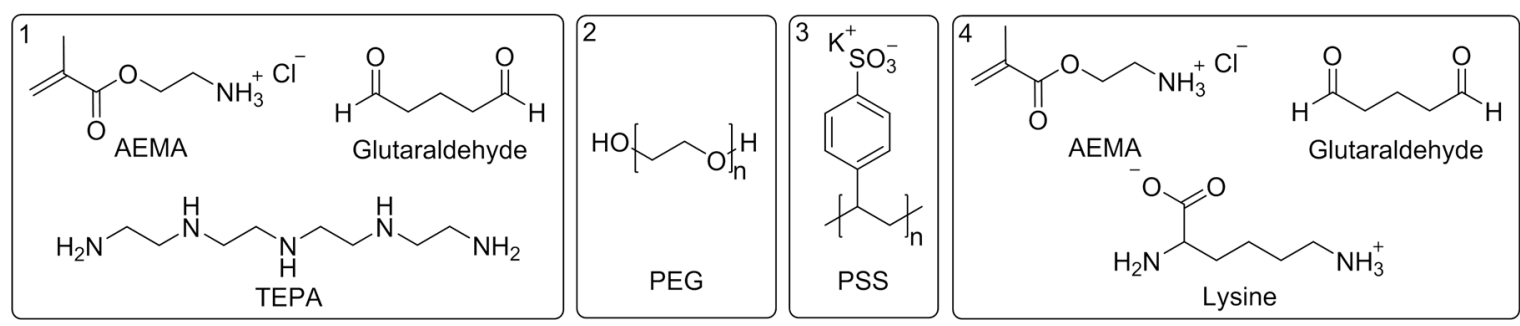

Figure 2. Modification reagents for (1) polyvinylidene fluoride-tetraethylenepentamine (PVDF-TEPA); (2) PVDF-polyethylene glycol (PVDF-PEG); (3) PVDF-polystyrene sulfonate (PVDF-PSS); and (4) PVDF-Lysine.

The resulting structures after modification with TEPA and Lysine surface functionalities as well as detailed descriptions of the reaction mechanisms leading to the different modification are known from literature and are therefore presented in the Supplementary Material (Figures S3 and S4).

The influence of the chosen membrane modifications was evaluated by measurement of water contact angle, zeta potential, XPS, SEM, mercury porosimetry, and water permeation flux. The initial water contact angle of an untreated PVDF membrane was determined to be $132^{\circ}$ (see Table 2) and by monitoring the water contact angle within the first $15 \mathrm{~min}$ the absorption rate of the applied water drop could be calculated to be $1.6^{\circ} / \mathrm{min}$. In the case of PVDF-PSS and PVDF-Lysine the initial values slightly decreased to $121^{\circ}$ and $114^{\circ}$, showing the increased wettability and therefore the successful modification. An even better result was gained after modification with PEG. Here, the initial water contact angle was found to be $92^{\circ}$, and also the absorption rate was increased to $3.5^{\circ} / \mathrm{min}$, indicating a faster uptake of water compared to the pristine membrane. Finally, the PVDF-TEPA membrane with its unique dendrimer structure showed an initial contact angle of $25^{\circ}$. Here, the absorption of the water drop was too fast to determine the absorption rate $(<3 \mathrm{~s})$.

Zeta potential measurements of the different membranes are presented in Figure 3 . The pristine PVDF membrane shows a trend that is typical for uncharged polymeric materials without further modifications. Due to the adsorption of hydroxide ions originating from the self-ionization of water the membrane surface appears to be negatively charged reaching its isoelectric point at a $\mathrm{pH}$ of $3.0[50,51]$ and has a zeta potential of $-40 \mathrm{mV}$ at $\mathrm{pH} 7$.

A similar trend is found for the PVDF-PEG membrane, where the IEP can be calculated by extrapolation to be around 2.2. The present polyethylene glycol chains do not contain charged functional groups and the zeta potential of $-45 \mathrm{mV}$ at $\mathrm{pH} 7$ is explained by the adsorbed hydroxide 
ions. However, compared to the pristine membrane, the IEP is slightly more acidic, what can be explained by small amounts of negatively charged functional groups which are present on the membrane surface and originate either from oxidation during irradiation or from oxidized impurities in the used PEG [52,53]. Nevertheless, when compared to the PVDF-PSS membrane it becomes clear that no additional negatively charged groups were added to the membrane surface. Therefore, a modification with PEG can still be considered as neutral, because the shift of IEP introduced by the modification with PSS is fundamentally higher. Here, the IEP is neither recorded nor calculable by a polynomial fit using the existing data. However, the PVDF-PSS membrane has a highly negative zeta potential of around $-50 \mathrm{mV}$ over a broad range of $\mathrm{pH}$ indicating the presence of sulfonic acid group immobilized on the membrane surface.

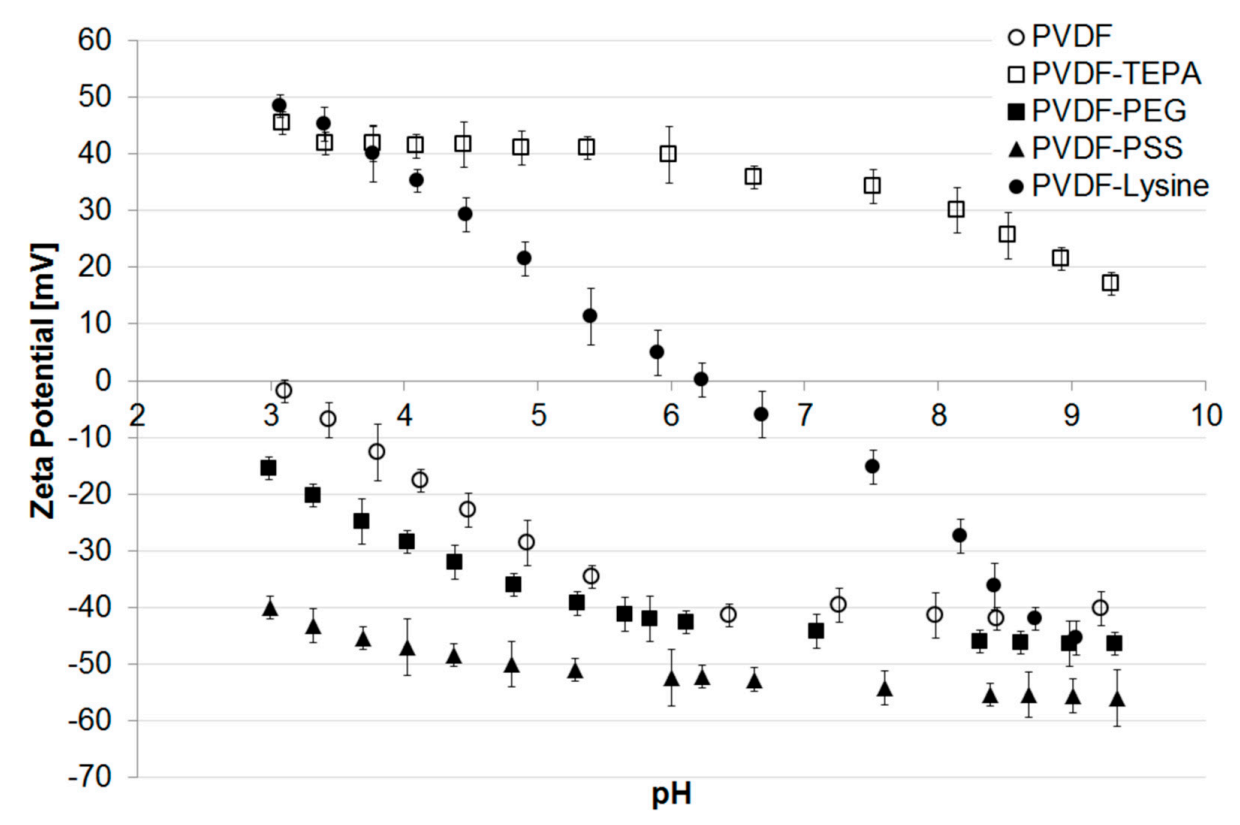

Figure 3. Zeta potential of different modified membranes vs. $\mathrm{pH}$.

The modification with a lysine containing structure, as shown in Figure S3.3 (Supplementary Material), leads to a membrane surface with both: acidic (carboxylic acid group) and alkaline (amino group) moieties: Accordingly, the PVDF-Lysine membrane's IEP was found to be at a nearly neutral $\mathrm{pH}$ of 6.2. Due to this, the membrane's zeta potential heavily depends on the $\mathrm{pH}$ of the surrounding solution: positive in the acidic range of $\mathrm{pH}$ (protonated amino groups) or negative in an alkaline environment (deprotonated acid groups).

The highest IEP amongst the prepared membranes can be reached with the PVDF-TEPA membrane. Due to amino functions present in the hyperbranched dendrimer structure, the modified membrane shows a high positive zeta potential of around $+40 \mathrm{mV}$ over a broad range of $\mathrm{pH}$. The IEP can be calculated to be around 10.3 .

\subsection{Membrane Fouling with PS Beads}

The main focus of this study was to investigate the influence of the membrane surface charge and its resulting electrostatic interactions on the fouling characteristics. However, the large number of different fouling reagents present in current literature lack in comparability. To solve this problem, this study uses monodisperse polystyrene beads (200 $\mathrm{nm}$ in diameter) with adjusted surface charges to describe the fouling that occurs or is inhibited by electrostatic interactions of the membrane's surface charge with charges present at the fouling reagent.

Figure 4a shows the fouling characteristics of different membranes towards anionic or cationic PS beads. Permeation flux and the relative concentration of polymer beads in the filtrate are plotted 
against the volume of PS bead suspension passed though the membrane. For a better overview, we focus on specific examples. However, the complete overview of fouling experiments is given in the Supplementary Material.

In the case of fouling with anionic beads, PVDF-PEG and PVDF-PSS showed the best anti-fouling properties. Their initial permeation flux of around $40 \mathrm{~mL} /\left(\mathrm{min} \cdot \mathrm{cm}^{2} \cdot\right.$ bar) neither decreased nor was a significant retention of PS beads detectable. Contrarily to this, intense fouling occurred using the pristine PVDF membrane as well as the PVDF-TEPA or PVDF-Lysine membrane, as shown in the Supplementary Material. For example the permeation flux of the pristine PVDF membrane drops to about $20 \%$ compared to its starting value after filtration of $140 \mathrm{~mL}$ of fouling suspension.
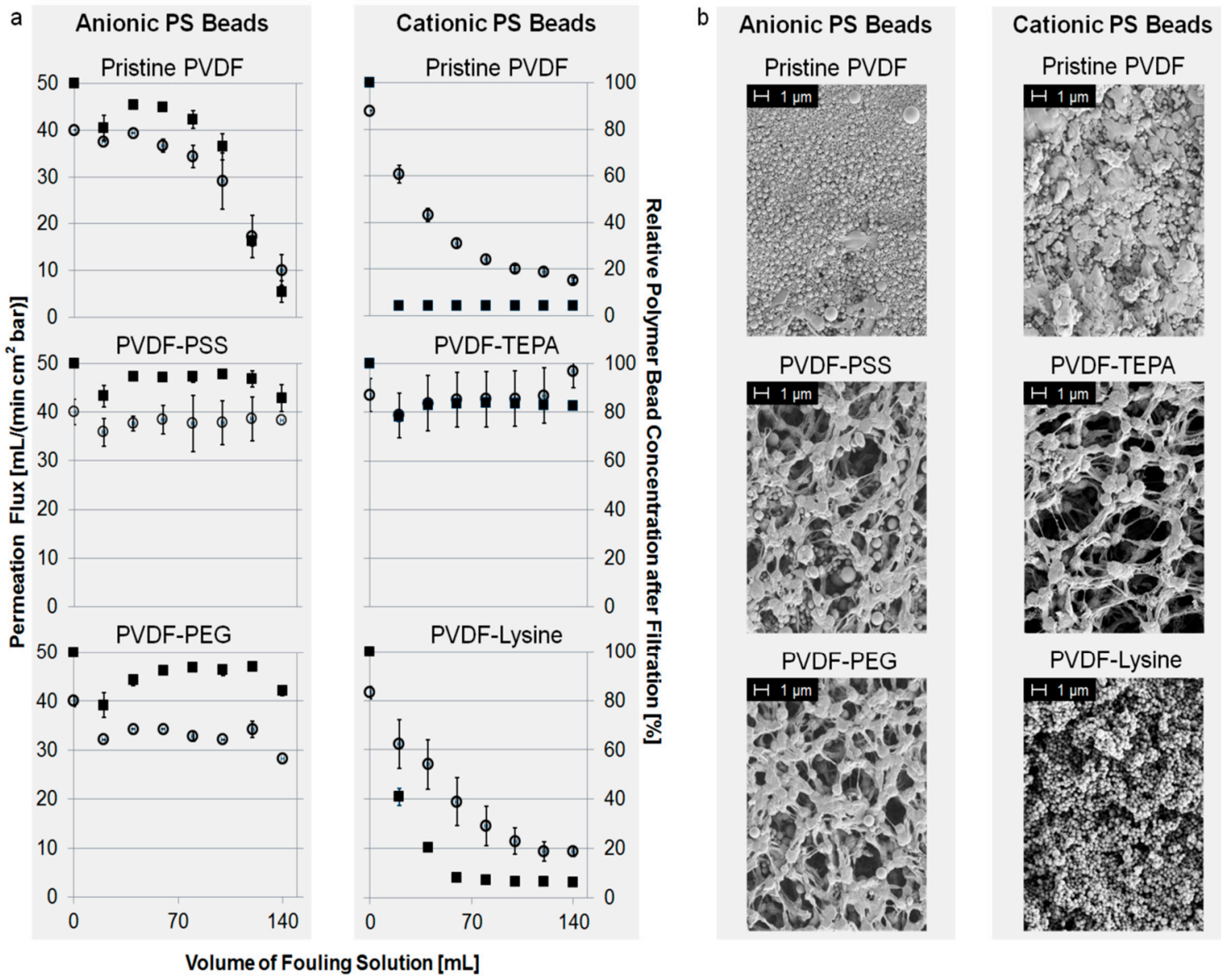

Figure 4. (a) Permeation flux of fouling suspension (left axis, open circles) and normalized concentration of PS beads in filtrate (right axis, filled squares) vs. volume of PS bead suspension; and (b) scanning electron microscopy (SEM) pictures of modified membranes after fouling.

Fouling with cationic PS beads can be prevented using the PVDF-TEPA membrane. Other membranes like the PVDF-Lysine membrane show strong and instantaneous fouling, and therefore, a drop in permeation flux down to $20 \%$ compared to its starting value. The results of the fouling experiments of PVDF-PSS and PVDF-PEG are shown in the Supplementary Materials.

Fouling with uncharged PS beads leads to pore blocking and permeation flux decline regarding the PVDF-TEPA, PVDF-Lysine and the pristine membrane. PVDF-PSS and PVDF-PEG instead show a good fouling resistance against the uncharged PS beads without permeation flux decline or bead retention. All results regarding fouling with uncharged PS beads are shown in the supplementary material.

While discussing the initial adsorption process during fouling of the various membranes the crucial forces that should be considered are hydrophobic and electrostatic interactions. In aqueous 
media hydrophobic interactions lead to a minimization of the interface between hydrophobic surfaces and surrounding water, which is also true for the adsorption of PS particles to a membrane surface. The process usually goes along with the release of water molecules originating from the hydration shells of the now interacting surfaces. Therefore, hydrophobic interactions are thermodynamically and entropically driven leading the system to a more stable state. Electrostatic forces on the other hand describe how charged objects interact, and therefore, if a repulsion or an attraction between membrane and particle is expected.

The pristine PVDF membrane is highly hydrophobic (initial water contact angle of $132^{\circ}$, as shown in Table 2) and possesses only adsorbed hydroxide ions as charged groups. The results show that the pristine membrane is fouled by all applied bead species. One would expect that there should be at least a slight electrostatic repulsion between the membrane (adsorbed hydroxide ions) and the anionic PS beads (sulfonic acid groups) or between the membrane and the uncharged PS beads (adsorbed hydroxide ions, see Figure S5 in the Supplementary Material), but these interactions appear to be not dominant enough. Therefore, the driving force of the observed membrane fouling must be the far greater energetic advantage gained from adsorption of the applied PS beads minimizing the membrane water interface.

Table 2. Wettability characteristics of modified PVDF membranes.

\begin{tabular}{lccccc}
\hline Determined parameter & PVDF & PVDF-TEPA & PVDF-PEG & PVDF-PSS & PVDF-Lysine \\
\hline Initial water contact angle $\left(^{\circ}\right)$ & $132 \pm 1$ & $25 \pm 1$ & $92 \pm 6$ & $121 \pm 2$ & $114 \pm 5$ \\
Drop absorption rate * $\left({ }^{\circ} / \mathrm{min}\right)$ & $1.6 \pm 0.1$ & $* *$ & $3.5 \pm 0.1$ & $1.6 \pm 0.3$ & $1.8 \pm 0.3$ \\
\hline & * Calculated based on time resolved contact angle measurements over 15 min; ${ }^{* *}$ Instantaneous absorption: \\
calculation impossible.
\end{tabular}

In the case of the hydrophilic PVDF-TEPA membrane (initial water contact angle of $25^{\circ}$, as shown in Table 2), the present amino functions are protonated at the working $\mathrm{pH}$ of 7 [54], giving the membrane a highly positive charge $(+40 \mathrm{mV})$. Consequently, anti-fouling properties towards cationic PS particles and instantaneous adsorption of anionic and uncharged PS beads are observed (Figure 4 and Figure S6 in the Supplementary Material). The latter is unexpected considering the high hydrophilicity of the membrane and the resulting water film, which should be able to prevent adsorption to the membrane surface. An explanation is provided by considering electrostatic interactions: cationic particles (protonated amino groups) are repelled by the positively charged PVDF-TEPA membrane (protonated amino groups), while uncharged PS beads (adsorbed hydroxide ions) and negatively charged (sulfonic acid groups) are attracted. Therefore, the driving forces for fouling of this membrane are electrostatic interactions, which are even capable to overcome the high hydrophilicity and, thus, the existing water film on the membrane surface.

The PVDF-PEG membrane is negatively charged $(-45 \mathrm{mV})$ at the working $\mathrm{pH}$ of 7 and more hydrophilic (initial water contact angle of $92^{\circ}$ as shown in Table 2) compared to the pristine membrane, leading to formation of a water film on the membrane surface. The experiments reveal good fouling resistance for uncharged and anionic PS beads, but fouling occurs for cationic PS beads (Figure 4 and Figure S7 in the Supplementary Material). Due to the high hydrophilicity of the membrane fouling would be generally unexpected but it occurs for cationic PS beads. Contrarily, the observed fouling tendencies are consistent with what is expected by considering electrostatic interactions: repulsion occurs between the membrane (adsorbed hydroxide ions) and uncharged (adsorbed hydroxide ions) or anionic PS beads (sulfonic acid groups), while cationic particles (protonated amino groups) are attracted. However, in the case of the pristine PVDF membrane these electrostatic interactions were shown to be not efficient. Only by discussing the addition of both effects (hydrophilicity and electrostatic repulsion), the anti-fouling characteristic towards uncharged and anionic PS beads can be explained. Regarding the fouling tendency of cationic beads, electrostatic 
attraction and the membrane's hydrophilicity are in opposition to each other and result in membrane fouling due to the more dominant electrostatic forces.

Compared to the pristine membrane the PVDF-PSS membrane is slightly more hydrophilic (initial water contact angle of $121^{\circ}$, as shown in Table 2$)$ and is highly negatively charged $(-52 \mathrm{mV})$ over a broad range of $\mathrm{pH}$. Contrarily to the experimental results of the PVDF-TEPA membrane cationic PS beads are adsorbed while anionic and uncharged PS particles do not result in membrane fouling (Figure 4 and Figure S8 in the Supplementary Material). Based on hydrophilicity a general anti-fouling would be expected but cationic PS particles still adsorb to the membrane surface. The experimental results can only be explained by considering additional electrostatic interactions: electrostatic repulsion occurs between the membrane (sulfonic acid groups) and uncharged (adsorbed hydroxide ions) or anionic PS beads (sulfonic acid groups), while cationic particles (protonated amino groups) are attracted. In the case of electrostatic repulsion, the membrane's hydrophilicity might also help to prevent fouling of uncharged or anionic beads. However, the fact that electrostatic attraction of cationic particles overcomes the membrane's hydrophilicity resulting in fouling, leads to the conclusion that electrostatic interactions are the main driving forces for fouling of this membrane, especially when compared to the PVDF-PEG membrane, where both effects are needed to explain the results.

The PVDF-Lysine membrane is also more hydrophilic compared to pristine membrane (initial water contact angle of $114^{\circ}$, as shown in Table 2) and due to the zwitterionic structure, consisting of both: negatively charged (carboxylic acid) and positively charged groups (amino groups), the IEP was found to be at a nearly neutral $\mathrm{pH}$ of 6.2. Therefore, the membrane exhibits a zeta potential of nearly zero $(-8 \mathrm{mV})$ at the working $\mathrm{pH}$ of 7 . The results show that the membrane, just like the pristine PVDF membrane, is fouled by all applied bead species (Figure 4 and Figure S9 in the Supplementary Material). This was unexpected because zwitterionic structures are reported to have a generally good fouling resistance [24], and also the surface is more hydrophilic compared to pristine membrane and, therefore, the existing water film should help to prevent membrane fouling. Electrostatic interactions can be excluded because of the zeta potential of nearly zero. A possibility to explain the found fouling results would be that the membrane surface is in an energetically unfavorable state due to the missing zeta potential and, thus, regains stability by adsorbing particles from the suspension. This effect is known for particle suspensions, which tend to agglomerate when the zeta potential drops below a certain level [55]. The authors suggest that a comparable effect occurs for the PVDF-Lysine membrane at $\mathrm{pH} 7$ showing, again, the importance of zeta potential and electrostatic interactions to explain membrane fouling.

SEM pictures confirm the fouling experiments as shown in Figure $4 \mathrm{~b}$. The degree of PS beads adsorbed to the membrane surface is in agreement with the data obtained from the fouling test described above. In the case of fouling with anionic or uncharged PS beads, PVDF-PEG and PVDF-PSS show good anti-fouling properties. Contrarily, intense fouling occurred for PVDF-TEPA, PVDF-Lysine and the pristine membrane using anionic or uncharged PS beads. Fouling with cationic PS beads could only be prevented by the PVDF-TEPA membrane; all other membranes show intense pore blocking. The complete overview of SEM pictures after fouling is given in the supplementary material.

The fouling characteristics of the PVDF-Lysine membrane at $\mathrm{pH} 7$ were unexpectedly poor: all applied bead species were adsorbed to the membrane surface. When the zeta potential of nearly zero is considered as the reason for this fouling tendency a change in $\mathrm{pH}$ of the surrounding solution and, therefore, of the membrane's zeta potential should result in a different fouling characteristic. Thus, some further experiments were conducted with fouling solutions at $\mathrm{pH} 4$ and 9 since the zeta potential (Figure 3) of the PVDF-Lysine membrane was determined to be positive at low $\mathrm{pH}(+40 \mathrm{mV}$ at $\mathrm{pH} 4)$ and negative at high $\mathrm{pH}(-45 \mathrm{mV}$ at $\mathrm{pH} 9)$.

As shown in Figure 5a, fouling of cationic PS beads can be prevented by shifting the $\mathrm{pH}$ to a value of 4, but uncharged PS beads still tend to foul the PVDF-Lysine membrane. The reason 
for this is the highly positive charge $(+40 \mathrm{mV})$ present at the PVDF-Lysine membrane's surface at $\mathrm{pH} 4$, which electrostatically repels the positively charged PS beads but attracts the uncharged PS beads (adsorbed hydroxide ions) regardless of the membrane's hydrophilicity. Contrarily, fouling of uncharged and negatively charged PS beads can be prevented at a $\mathrm{pH}$ of 9, where the PVDF-Lysine membrane possesses a highly negative potential of $-45 \mathrm{mV}$ and, therefore, inhibits interactions between the membrane and the particles by electrostatic repulsion. The hydrophilic properties of the PVDF-Lysine membrane may also help to prevent fouling of anionic and uncharged PS beads at $\mathrm{pH} \mathrm{9,} \mathrm{however,} \mathrm{the} \mathrm{fact} \mathrm{that} \mathrm{electrostatic} \mathrm{attraction} \mathrm{of} \mathrm{uncharged} \mathrm{particles} \mathrm{at} \mathrm{pH} 4$ overcomes the membrane's hydrophilicity leads to the conclusion that electrostatic interactions are the main driving forces for fouling of this membrane. The corresponding SEM pictures (Figure 5b) confirm the results of the fouling experiments.

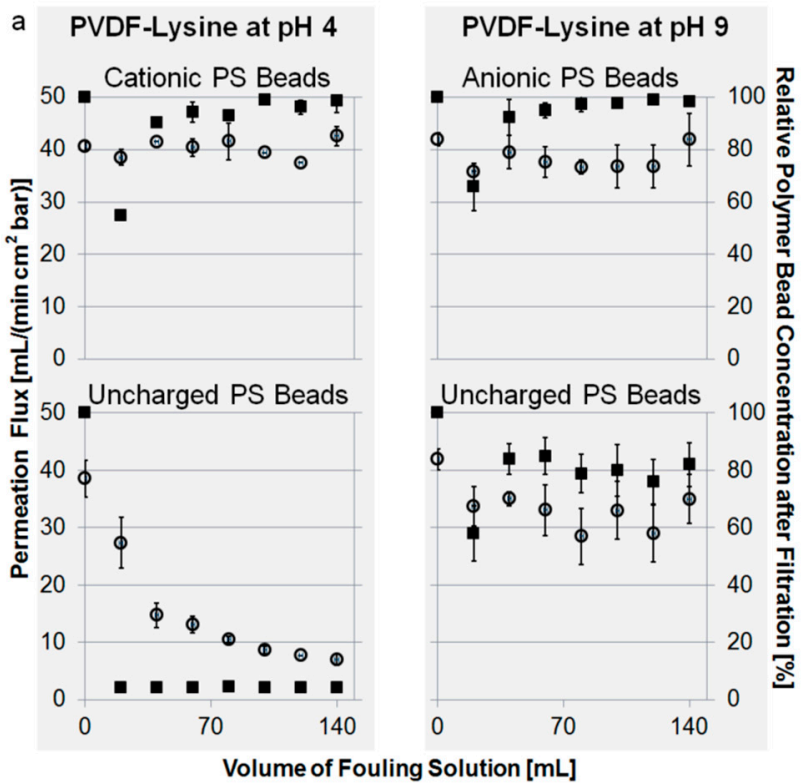

b PVDF-Lysine at $\mathrm{pH} 4$

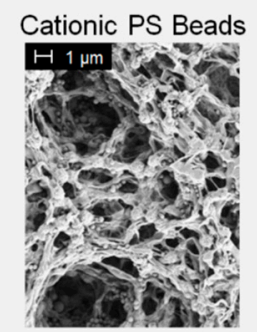

Uncharged PS Beads

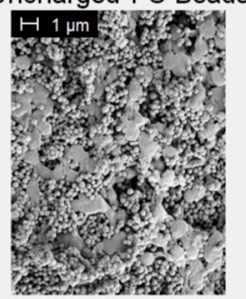

PVDF-Lysine at $\mathrm{pH} 9$

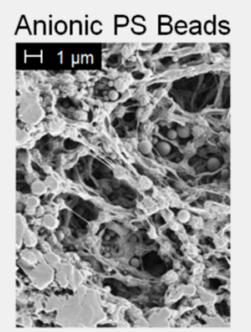

Uncharged PS Beads

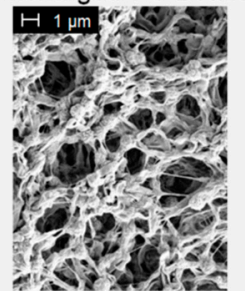

Figure 5. (a) Permeation flux of fouling suspension (left axis, open circles) and normalized concentration of PS beads in filtrate (right axis, filled squares) vs. volume of PS bead suspension; and (b) SEM pictures of the top side of modified membranes.

\section{Conclusions}

In this study, we demonstrated that membrane fouling of microfiltration membranes significantly depends on the electrostatic interactions between the membrane surface and the fouling reagent. To enable a comparative study, we developed a test system using monodisperse polystyrene beads with a size of around $200 \mathrm{~nm}$ and specific surface charges (positive, uncharged, negative). The used PVDF membranes (pore size $0.45 \mu \mathrm{m}$ ) were modified by electron beam irradiation with different reagents introducing specific charged groups. Our study revealed that:

- Highly hydrophobic systems like the pristine PVDF membrane are mainly fouled due to hydrophobic interactions while electrostatic interactions are less important.

- Electrostatic attractive interactions are the driving forces of fouling when the membrane surface and the fouling reagent are oppositely charged (e.g., PVDF-PSS + cationic PS beads or PVDF-TEPA + anionic PS beads).

- Electrostatic repulsive interactions are the dominating forces for obtaining fouling resistance when the membrane surface and the fouling reagent are evenly charged (e.g., PVDF-PSS + anionic PS beads, PVDF-TEPA + cationic PS beads, PVDF-Lysine + anionic PS beads at $\mathrm{pH} 9$ or PVDF-Lysine + cationic PS beads at $\mathrm{pH} 4$ ). 
- Hydrophilized, non-charged membranes like the PVDF-PEG membrane show fouling characteristics that can only be explained when both hydrophilicity and electrostatic interactions are considered.

- A zeta potential of nearly zero (PVDF-Lysine membrane at $\mathrm{pH}$ 7) results in membrane fouling that can be prevented by, e.g., changes in $\mathrm{pH}$.

These results indicate that fouling significantly depends on a membrane's zeta potential but also on the hydrophilicity. To prevent fouling due to hydrophobic interactions, membranes should possess a hydrophilic surface, which assures a covering water film on the entire membrane surface. However, if charged fouling reagents are present, electrostatic repulsion is required to prevent fouling. This leads to the conclusion that both zeta potential and water contact angle measurements are required to characterize a membrane and predict its fouling tendencies. The driving force of fouling can be determined by using the invented test system with differently charged PS beads.

Supplementary Materials: The supplementary materials is available online at: www.mdpi.com/2073-4360/ 7/10/1496/s1.

Acknowledgments: The authors thank Nadja Schönherr for measuring particle size, zeta potential and isoelectric point of the PS beads. Financial support by the Federal State of Germany and the Free State of Saxony is gratefully acknowledged.

Author Contributions: Agnes Schulze and Daniel Breite conceived and designed the experiments; Daniel Breite performed the experiments; Marco Went performed the porosimetry measurements; Andrea Prager performed the SEM and XPS measurements; and Daniel Breite wrote the paper.

Conflicts of Interest: The authors declare no conflict of interest.

\section{References}

1. Haynes, C.A.; Norde, W. Structures and stabilities of adsorbed proteins. J. Colloid Interface Sci. 1995, 169, 313-328. [CrossRef]

2. Wahlgren, M.; Arnebrant, T. Protein adsorption to solid surfaces. Trends Biotechnol. 1991, 9, $201-208$. [CrossRef]

3. Khulbe, K.C.; Feng, C.; Matsuura, T. The art of surface modification of synthetic polymeric membranes. J. Appl. Polym. Sci. 2010, 115, 855-895. [CrossRef]

4. Liu, S.X.; Kim, J.T.; Kim, S. Effect of polymer surface modification on polymer-protein interaction via hydrophilic polymer grafting. J. Food Sci. 2008, 73, E143-E150. [CrossRef] [PubMed]

5. Chu, P.K.; Chen, J.Y.; Wang, L.P.; Huang, N. Plasma-surface modification of biomaterials. Mater. Sci. Eng. 2002, 36, 143-206. [CrossRef]

6. Van der Bruggen, B. Chemical modification of polyethersulfone nanofiltration membranes: A review. J. Appl. Polym. Sci. 2009, 114, 630-642. [CrossRef]

7. Saxena, N.; Prabhavathy, C.; de, S.; DasGupta, S. Flux enhancement by argon-oxygen plasma treatment of polyethersulfone membranes. Sep. Purif. Technol. 2009, 70, 160-165. [CrossRef]

8. Chang, Y.; Ko, C.-Y.; Shih, Y.-J.; Quémener, D.; Deratani, A.; Wei, T.-C.; Wang, D.-M.; Lai, J.-Y. Surface grafting control of pegylated poly(vinylidene fluoride) antifouling membrane via surface-initiated radical graft copolymerization. J. Membr. Sci. 2009, 345, 160-169. [CrossRef]

9. Du, J.R.; Peldszus, S.; Huck, P.M.; Feng, X. Modification of poly(vinylidene fluoride) ultrafiltration membranes with poly(vinyl alcohol) for fouling control in drinking water treatment. Water Res. 2009, 43, 4559-4568. [CrossRef] [PubMed]

10. Chen, H.; Belfort, G. Surface modification of poly(ether sulfone) ultrafiltration membranes by low-temperature plasma-induced graft polymerization. J. Appl. Polym. Sci. 1999, 72, 1699-1711. [CrossRef]

11. Ulbricht, M. Advanced functional polymer membranes. Polymer 2006, 47, 2217-2262. [CrossRef]

12. Shi, Q.; Su, Y.; Chen, W.; Peng, J.; Nie, L.; Zhang, L.; Jiang, Z. Grafting short-chain amino acids onto membrane surfaces to resist protein fouling. J. Membr. Sci. 2011, 366, 398-404. [CrossRef]

13. Schulze, A.; Marquardt, B.; Kaczmarek, S.; Schubert, R.; Prager, A.; Buchmeiser, M.R. Electron beam-based functionalization of poly(ethersulfone) membranes. Macromol. Rapid Commun. 2010, 31, 467-472. [CrossRef] [PubMed] 
14. Schulze, A.; Marquardt, B.; Went, M.; Prager, A.; Buchmeiser, M.R. Electron beam-based functionalization of polymer membranes. Water Sci. Technol. 2012, 65, 574-580. [CrossRef] [PubMed]

15. Boulares-Pender, A.; Thomas, I.; Prager, A.; Schulze, A. Surface modification of polyamide and poly(vinylidene fluoride) membranes. J. Appl. Polym. Sci. 2013, 128, 322-331. [CrossRef]

16. Khulbe, K.C.; Matsuura, T. Characterization of PPO membranes by oxygen plasma etching, gas separation and atomic force microscopy. J. Membr. Sci. 2000, 171, 273-284. [CrossRef]

17. Asfardjani, K.; Segui, Y.; Aurelle, Y.; Abidine, N. Effect of plasma treatments on wettability of polysulfone and polyetherimide. J. Appl. Polym. Sci. 1991, 43, 271-281. [CrossRef]

18. Buonomenna, M.G.; Lopez, L.C.; Favia, P.; d'Agostino, R.; Gordano, A.; Drioli, E. New pvdf membranes: The effect of plasma surface modification on retention in nanofiltration of aqueous solution containing organic compounds. Water Res. 2007, 41, 4309-4316. [CrossRef] [PubMed]

19. Vandencasteele, N.; Reniers, F. Plasma-modified polymer surfaces: Characterization using XPS. J. Electron Spectrosc. Relat. Phenom. 2010, 178-179, 394-408. [CrossRef]

20. Kull, K.R.; Steen, M.L.; Fisher, E.R. Surface modification with nitrogen-containing plasmas to produce hydrophilic, low-fouling membranes. J. Membr. Sci. 2005, 246, 203-215. [CrossRef]

21. Ingemar, L. Surface physics and biological phenomena. Phys. Scr. 1983, T4, 5-13.

22. Kumar, M.; Ulbricht, M. Novel ultrafiltration membranes with adjustable charge density based on sulfonated poly(arylene ether sulfone) block copolymers and their tunable protein separation performance. Polymer 2014, 55, 354-365. [CrossRef]

23. Xiao, K.; Wang, X.; Huang, X.; Waite, T.D.; Wen, X. Combined effect of membrane and foulant hydrophobicity and surface charge on adsorptive fouling during microfiltration. J. Membr. Sci. 2011, 373, 140-151. [CrossRef]

24. Hadidi, M.; Zydney, A.L. Fouling behavior of zwitterionic membranes: Impact of electrostatic and hydrophobic interactions. J. Membr. Sci. 2014, 452, 97-103. [CrossRef]

25. Zhang, Q.; Vecitis, C.D. Conductive CNT-PVDF membrane for capacitive organic fouling reduction. J. Membr. Sci. 2014, 459, 143-156. [CrossRef]

26. Dudchenko, A.V.; Rolf, J.; Russell, K.; Duan, W.; Jassby, D. Organic fouling inhibition on electrically conducting carbon nanotube-polyvinyl alcohol composite ultrafiltration membranes. J. Membr. Sci. 2014, 468, 1-10. [CrossRef]

27. Childress, A.E.; Elimelech, M. Relating nanofiltration membrane performance to membrane charge (electrokinetic) characteristics. Environ. Sci. Technol. 2000, 34, 3710-3716. [CrossRef]

28. Hong, S.; Elimelech, M. Chemical and physical aspects of natural organic matter (NOM) fouling of nanofiltration membranes. J. Membr. Sci. 1997, 132, 159-181. [CrossRef]

29. Young, T. An essay on the cohesion of fluids. Philos. Trans. R. Soc. Lond. 1805, 95, 65-87. [CrossRef]

30. Arkhangelsky, E.; Levitsky, I.; Gitis, V. Electrostatic repulsion as a mechanism in fouling of ultrafiltration membranes. Water Sci. Technol. 2008, 58, 1955-1961. [CrossRef] [PubMed]

31. Han, M.J.; Baroña, G.N.B.; Jung, B. Effect of surface charge on hydrophilically modified poly(vinylidene fluoride) membrane for microfiltration. Desalination 2011, 270, 76-83. [CrossRef]

32. Lamminen, M.O.; Walker, H.W.; Weavers, L.K. Effect of fouling conditions and cake layer structure on the ultrasonic cleaning of ceramic membranes. Sep. Sci. Technol. 2006, 41, 3569-3584. [CrossRef]

33. Wang, Y.; Wicaksana, F.; Tang, C.Y.; Fane, A.G. Direct microscopic observation of forward osmosis membrane fouling. Environ. Sci. Technol. 2010, 44, 7102-7109. [CrossRef] [PubMed]

34. Ma, H.; Hakim, L.F.; Bowman, C.N.; Davis, R.H. Factors affecting membrane fouling reduction by surface modification and backpulsing. J. Membr. Sci. 2001, 189, 255-270. [CrossRef]

35. Bondy, C.M.; Santeufemio, C. Analysis of fouling within microporous membranes in biopharmaceutical applications using latex microsphere suspensions. J. Membr. Sci. 2010, 349, 12-24. [CrossRef]

36. Teychene, B.; Loulergue, P.; Guigui, C.; Cabassud, C. Development and use of a novel method for in line characterisation of fouling layers electrokinetic properties and for fouling monitoring. J. Membr. Sci. 2011, 370, 45-57. [CrossRef]

37. Abdelrasoul, A.; Doan, H.; Lohi, A.; Cheng, C.-H. Modeling of fouling and foulant attachments on heterogeneous membranes in ultrafiltration of latex solution. Sep. Purif. Technol. 2014, 135, 199-210. [CrossRef] 
38. Gordano, A.; Arcella, V.; Drioli, E. New HYFLON AD composite membranes and AFM characterization. Desalination 2004, 163, 127-136. [CrossRef]

39. Lamminen, M.O.; Walker, H.W.; Weavers, L.K. Mechanisms and factors influencing the ultrasonic cleaning of particle-fouled ceramic membranes. J. Membr. Sci. 2004, 237, 213-223. [CrossRef]

40. Lamminen, M.O.; Walker, H.W.; Weavers, L.K. Cleaning of particle-fouled membranes during cross-flow filtration using an embedded ultrasonic transducer system. J. Membr. Sci. 2006, 283, 225-232. [CrossRef]

41. Adamczyk, Z.; Warszyhski, P. Role of electrostatic interactions in particle adsorption. Adv. Colloid Interface Sci. 1996, 63, 41-149. [CrossRef]

42. Adamczyk, Z. Particle adsorption and deposition: Role of electrostatic interactions. Adv. Colloid Interface Sci. 2003, 100, 267-347. [CrossRef]

43. Henry, C.; Minier, J.-P.; Lefèvre, G. Towards a description of particulate fouling: From single particle deposition to clogging. Adv. Colloid Interface Sci. 2012, 185, 34-76. [CrossRef] [PubMed]

44. Lanteri, Y.; Fievet, P.; Magnenet, C.; Déon, S.; Szymczyk, A. Electrokinetic characterisation of particle deposits from streaming potential coupled with permeate flux measurements during dead-end filtration. J. Membr. Sci. 2011, 378, 224-232. [CrossRef]

45. Inukai, S.; Tanma, T.; Orihara, S.; Konno, M. A simple method for producing micron-sized, highly monodisperse polystyrene particles in aqueous media: Effects of impeller speed on particle size distribution. Chem. Eng. Res. Des. 2001, 79, 901-905. [CrossRef]

46. Duracher, D.; Sauzedde, F.; Elaissari, A.; Perrin, A.; Pichot, C. Cationic amino-containing $\mathrm{N}$-isopropyl-acrylamide-styrene copolymer latex particles: 1-Particle size and morphology vs. polymerization process. Colloid Polym. Sci. 1998, 276, 219-231. [CrossRef]

47. Yoon, S.Y.; Park, Y.-S.; Lee, J.S. Controlled synthesis of spherical polystyrene beads and their template-assisted manual assembly. Bull. Korean Chem. Soc. 2014, 35, 2281-2284. [CrossRef]

48. Schulze, A.; Maitz, M.F.; Zimmermann, R.; Marquardt, B.; Fischer, M.; Werner, C.; Went, M.; Thomas, I. Permanent surface modification by electron-beam-induced grafting of hydrophilic polymers to PVDF membranes. RSC Adv. 2013, 3, 22518-22526. [CrossRef]

49. Boulares-Pender, A.; Prager, A.; Elsner, C.; Buchmeiser, M.R. Surface-functionalization of plasma-treated polystyrene by hyperbranched polymers and use in biological applications. J. Appl. Polym. Sci. 2009, 112, 2701-2709. [CrossRef]

50. Zimmermann, R.; Dukhin, S.; Werner, C. Electrokinetic measurements reveal interfacial charge at polymer films caused by simple electrolyte ions. J. Phys. Chem. B 2001, 105, 8544-8549. [CrossRef]

51. Zimmermann, R.; Freudenberg, U.; Schweiß, R.; Küttner, D.; Werner, C. Hydroxide and hydronium ion adsorption-A survey. Curr. Opin. Colloid Interface Sci. 2010, 15, 196-202. [CrossRef]

52. Ray, W.J., Jr.; Puvathingal, J.M. A simple procedure for removing contaminating aldehydes and peroxides from aqueous solutions of polyethylene glycols and of nonionic detergents that are based on the polyoxyethylene linkage. Anal. Biochem. 1985, 146, 307-312. [CrossRef]

53. Jurnak, F. Effect of chemical impurities in polyethylene glycol on macromolecular crystallization. J. Cryst. Growth 1986, 76, 577-582. [CrossRef]

54. Burke, S.E.; Barrett, C.J. Acid-base equilibria of weak polyelectrolytes in multilayer thin films. Langmuir 2003, 19, 3297-3303. [CrossRef]

55. Jiang, J.; Oberdörster, G.; Biswas, P. Characterization of size, surface charge, and agglomeration state of nanoparticle dispersions for toxicological studies. J. Nanopart. Res. 2008, 11, 77-89. [CrossRef]

(C) 2015 by the authors; licensee MDPI, Basel, Switzerland. This article is an open access article distributed under the terms and conditions of the Creative Commons by Attribution (CC-BY) license (http://creativecommons.org/licenses/by/4.0/). 